\title{
Water Maser Emission Around Low/Intermediate Mass Evolved Stars
}

\author{
M. L. Leal-Ferreira ${ }^{1}$, W. H. T. Vlemmings ${ }^{2}$, P. J. Diamond ${ }^{3}$, \\ A. Kemball ${ }^{4}$, N. Amiri ${ }^{5,6}$ and J.-F. Desmurs ${ }^{7}$ \\ ${ }^{1}$ Argelander Institute für Astronomie, Uni-Bonn, Auf dem Hügel 71, 53121 Bonn, Germany \\ email: ferreira@astro.uni-bonn.de \\ ${ }^{2}$ OSO (Sweden), ${ }^{3} \mathrm{JBCA}$ (UK), ${ }^{4}$ University of Illinois (USA), ${ }^{5} \mathrm{JIVE}$ (Netherlands), ${ }^{6}$ Leiden \\ Observatory (Netherlands), ${ }^{7}$ OAN (Spain)
}

\begin{abstract}
We present results of Very Long Baseline Array (VLBA) polarimetric $22 \mathrm{GHz} \mathrm{H}_{2} \mathrm{O}$ maser observations of a number of low/intermediate mass evolved stars. We observed 3 Miras (Ap Lyn, IK Tau and IRC+60370), 1 semi-regular variable (RT Vir) and 1 pPN (OH231.8+4.2). Circular polarization is detected in the $\mathrm{H}_{2} \mathrm{O}$ maser region of $\mathrm{OH} 231.8+4.2$ and we infer a magnetic field of $\left|B_{\|}\right|=\sim 45 \mathrm{mG}$. This implies an extrapolated magnetic field of $\sim 2.5 \mathrm{G}$ on the surface of the central star. The preliminary results on RT Vir and IRC +60370 also indicate the first detection of weak $\mathrm{H}_{2} \mathrm{O}$ maser linear polarization.
\end{abstract}

Keywords. masers, magnetic fields, polarization, stars: AGB and post-AGB

\section{Introduction}

During the transition from an AGB star to a planetary nebula $(\mathrm{PN})$, most low and intermediate mass stars lose their spherical symmetry. Magnetic fields are one of the candidates that can play a role in shaping asymmetrical PNe. However, magnetic field observations around evolved stars are still rare. We observed $22.235080 \mathrm{GHz} \mathrm{H}_{2} \mathrm{O}$ masers around 5 evolved stars with the goal of measuring linear and circular polarization. The results may allow us to infer the magnetic fields properties around these 5 stars.

\section{Results}

\subsection{OH231.8+4.2 / Rotten Egg Nebula / Calabash Nebula (Leal-Ferreira et al. 2012)}

We detected $30 \mathrm{H}_{2} \mathrm{O}$ masers around $\mathrm{OH} 231.8+4.2$. With respect to the central star position, 20 masers are located on the north (NReg), and 10 on the south (SReg). We compared our detections with those of Desmurs et al. (2007), and found that the offset between the mean position of the detections of Desmurs et al. (2007) and ours is 14.4 mas. Taking a distance $\mathrm{d}=1540 \mathrm{pc}$ (Choi et al. 2012) and $i=36^{\circ}$ (Kastner et al. 1992; Shure et al. 1995), the separation velocity between the masers in the 2 regions is $21 \pm 11 \mathrm{~km} / \mathrm{s}$. The masers appear to be moving in the direction of the nebula jet, albeit at much lower velocity. This could indicate that they arise in a turbulent material entrained by the jet.

We found linear polarization for 3 features (all in SReg; Fig. 1). The high scatter between the linear polarization vectors can be caused by turbulence or, in case of a toroidal field, it could represent the tangent points of the field lines. We also found circular polarization on the 2 strongest masers; one in each region (Fig. 1). From these results, we inferred $\left|B_{\|}\right|_{N R e g}=44 \pm 7 \mathrm{mG}$ and $\left|B_{\|}\right|_{S R e g}=-29 \pm 21 \mathrm{mG}$. Although the morphology of the field is still not determined, the strength of the field on the surface of the star (with a typical radius of $1 \mathrm{AU})$ is $\sim 2.5 \mathrm{G}$ if we assume a toroidal magnetic field $(\mathrm{B} \propto 1 / \mathrm{r})$. 

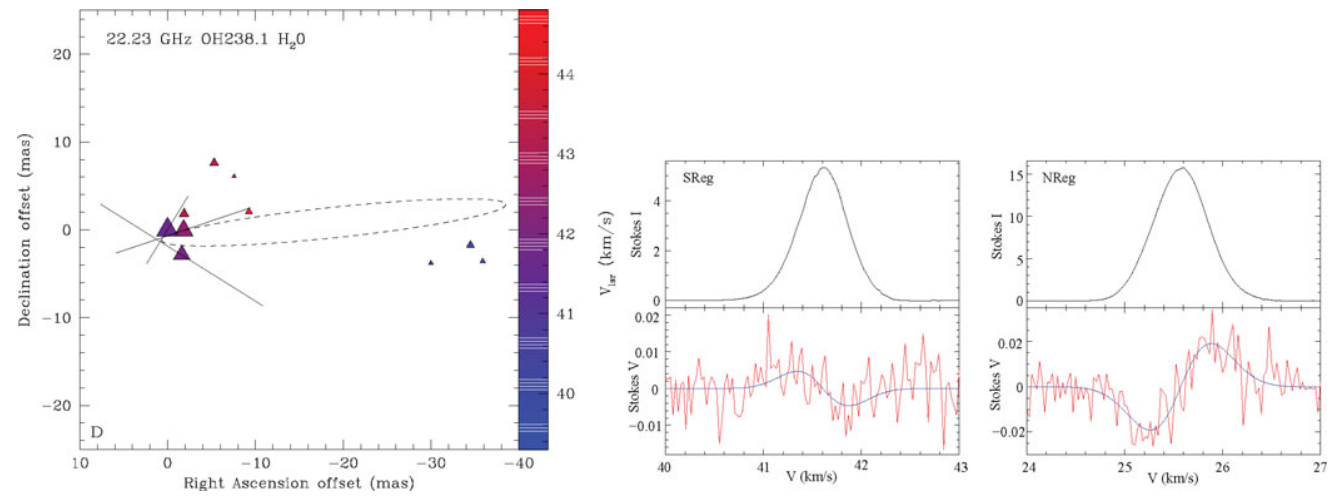

Figure 1. Left: Maser features observed in the region (SReg) around the Southern jet of $\mathrm{OH} 231.8+4.2$. The sizes of the triangles are scaled by their fluxes, and the colors follow the velocity scale. The vectors indicate the linear polarization directions, and the detached ellipse is a potential field morphology. Middle and right: I (top) and V (bottom) spectra of the brightest features we detected in the SReg (middle) and in the NReg (right) of OH231.8+4.2. The S-shape solid line is the best fit derivative of the total power spectrum.

\subsection{RT Vir, AP Lyn, IK Tau and IRC+60370}

We did not detect any maser emission around AP Lyn. Several masers were found around RT Vir, IK Tau and IRC+60370. Our preliminary results show that linear polarization between $\sim 0.5 \%$ and $\sim 0.8 \%$ is present around RT Vir and IRC+60370 (Fig 2). This is less than typically found in star forming regions and consistent with the previously found upper limits on the linear polarization in the $\mathrm{H}_{2} \mathrm{O}$ maser envelopes of Mira and supergiant stars (Vlemmings et al. 2005).
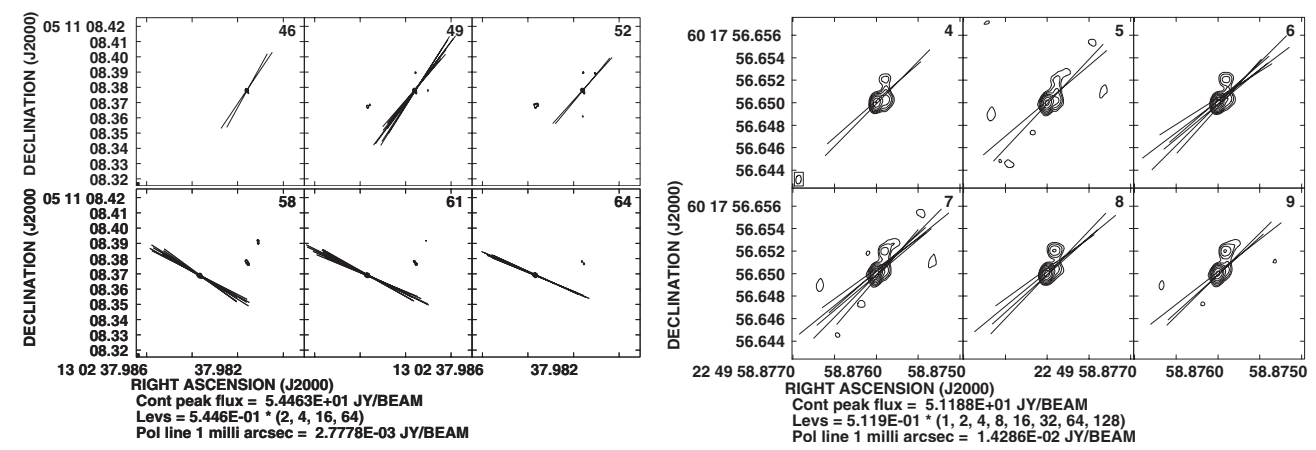

Figure 2. Contour maps of the maser spots of RT Vir (left) and IRC+60370 (right). For RT Vir we show one in every three channels in which linear polarization is present. For IRC +60370 we show 6 consecutive channels. The vectors indicate the linear polarization direction.

\section{References}

Choi Y. K., Brunthaler A., Menten K. M. \& Reid M. J. 2012, this proceedings

Desmurs J. -F., Alcolea J., Bujarrabal V., Sánchez Contreras C., et al. 2007, A\&A, 468, 189

Kastner J. H., Weintraub D. A., Zuckerman B., Becklin E. E., et al. 1992, ApJ, 398, 552

Leal-Ferreira M. L., Vlemmings W. H. T., Diamond P. J., et al. 2012, A\&\&A (accepted)

Shure M., Sellgren K., Jones T. J. \& Klebe D. 1995, AJ, 109, 721

Vlemmings, W. H. T., van Langevelde, H. J., \& Diamond, P. J. 2005, A\&A, 434, 1029 